\title{
An HIV-free generation: review of prevention strategies
}

\author{
I Moodley \\ Health Outcomes Research Unit, Discipline of Public Health Medicine, University of KwaZulu-Natal, South Africa \\ Corresponding author, email:moodleyi15@ukzn.ac.za
}

Globally South Africa has the largest HIV epidemic, with an estimated 7.7 million people living with HIV (PLHIV). The burden of HIV in South Africa varies by age, gender and key population groups. Prevalence is highest among adults between the ages of 15 and 49 years and is higher among females than males. The prevalence rate also varies by province with the highest rates observed in KwaZulu-Natal, Free State, Eastern Cape, Mpumalanga and Northwest.

While greater access to anti-retroviral treatment (ART) has significantly reduced the number of deaths and new infections, the number of new infections is still relatively high, highlighting the need for prevention.

Initial prevention strategies focus on behavioural change through increased education and awareness to ensure safer sex, mainly through peer education, condom distribution and voluntary medical male circumcision (VMMC), all of which are in place with varying degrees of success. More recently newer approaches are being considered such as pre-exposure prophylaxis (PrEP) and treatment as prevention (TasP). The major challenge is still behavioural change.

This review sets out to critically evaluate the current and newer approaches. Studies suggest that to achieve greater impact, rather than targeting the general population, prevention strategies should focus on vulnerable populations and in provinces with the highest HIV burden.

\section{Introduction}

In 2018, South Africa, which has the largest HIV epidemic in the world, had an estimated 7.7 million people, or $21 \%$ of those globally, living with HIV. ${ }^{1}$ The burden of HIV in South Africa varies greatly across the country by age, gender and for different key and vulnerable populations. The prevalence is highest among adults between the ages of 15 and 49 years with rates of $20.6 \%$, of which $26.3 \%$ is among females and $14.8 \%$ among males. This gender disparity in HIV prevalence is most marked among young adults. The HIV prevalence among 20 to 24 year olds is three times higher among females (15.6\%) than males (4.8\%). The prevalence rate also varies by province. The highest rates are observed in KwaZulu-Natal (27\%) with similar rates in Free State (25.5\%), Eastern Cape (25.2\%), Mpumalanga (22.8\%) and Northwest (22.7\%) with lower rates in the other provinces such as Western Cape (12.9\%), Northern Cape (13.9\%), Limpopo (17.2\%) and Gauteng (17.6\%). ${ }^{1,2}$

In 2017, 3.7 million people were taking ART for HIV, which was only $53 \%$ of those eligible for treatment. ${ }^{1}$ Of those on ART, $87.5 \%$ of those aged between 15 and 64 years are virally suppressed. Initially PLHIV with a CD count of $<350 / \mu$ l qualified for treatment with ART and, in 2011, 1.46 million were on ART. Currently anyone who is HIV positive is eligible for ART and 3.9 million have access. ${ }^{1}$

As a result of greater access to ART, the deaths due to HIV have dropped from 681434 in 2006 to an estimated 150376 in 2016. Furthermore, the number of new HIV infections fell during this period and a higher proportion of PLHIV were diagnosed and treated.
However, of concern is that in 2018 an estimated 240000 people became newly infected with HIV and there were 71000 AIDSrelated deaths. The highest incidence was among women (1.5\%) aged 15-24 years, declining slightly with age. Among PLHIV aged 15 to 64 years who know their HIV status, only $70.6 \%$ are on ART. ${ }^{1}$ It is therefore essential to prevent new infections through primary HIV-prevention strategies ${ }^{3}$ particularly among vulnerable populations. The Health Sector Prevention Strategy of $2016^{4}$ defines "vulnerable populations" as "groups of people that are particularly vulnerable to HIV infection under certain circumstances. These include young women and girls, orphaned and vulnerable children, people in prisons and detention centres, persons with disabilities, migrant and mobile workers, and seronegative partners in serodiscordant couples. Young women refers to girls between the ages of 15 and 19 years and young women between the ages of 20 and 29 years".

Initial prevention strategies focus on behavioural change, through increased education and awareness of HIV to ensure safer sex, mainly through peer education, condom distribution and VMMC all of which are in place with varying degrees of success. More recently newer approaches are being considered such as pre-exposure prophylaxis (PrEP) and treatment as prevention (TasP). This review sets out to critically evaluate the current and newer approaches.

\section{Review of strategies for prevention of new infections}

The South African government, through national HIV and AIDS control programmes, in collaboration with international development agencies, private voluntary organisations, and 
other non-governmental groups has dedicated numerous resources to developing low-cost interventions to arrest the spread of HIV and AIDS. ${ }^{4,5}$ Many different programmes have distributed AIDS information leaflets, as well as items such as badges and stickers. Messages informing people about the danger of AIDS are regularly broadcast on radio and television, published in newspapers, displayed on billboards and performed by local entertainers. These messages are reinforced through peer educators who visit local entertainment venues, STD clinics, and work sites to provide AIDS-prevention education and distribute free condoms. Millions of condoms are being made available at the workplace, airports, universities and other locations. ${ }^{2}$ When many of these interventions were first conceived, the expectation was that they would induce a sufficiently large behaviour response to contain the epidemic.

Since the rate of new infections is still relatively high, the question still remains as to how successful these efforts have in fact been at preventing new cases. While there are many limitations inherent in attempting to evaluate the effectiveness of interventions aimed at HIV prevention, data from various surveillance systems indicate that current interventions are probably not yet having a significant impact on the epidemic at the continental or even the local level. ${ }^{3,5}$ Despite the fact that levels of AIDS awareness are extremely high, ${ }^{3}$ getting people to change their behaviour is difficult. Factors such as denial, fear, stigma, external pressures, other priorities, or poverty can sometimes keep people from adopting healthier lifestyles.

It seems evident now that the focus of HIV-prevention programmes on preventing HIV transmission should be implemented through a combination of behavioural, biomedical and structural strategies selectively targeting the most vulnerable populations, particularly young women residing in provinces with the highest prevalence and incidence such as KwaZulu-Natal, Eastern Cape and Free State.

The current and newer strategies are critically reviewed with the view of identifying some of their successes and challenges.

\section{Peer educators}

A systematic review and meta-analysis of the effectiveness of peer education programmes as an HIV-prevention strategy in developing countries, provided evidence that peer education programmes are moderately effective at improving knowledge and behavioural outcomes. ${ }^{6}$ However, a limitation identified in the review and analysis was that many of the study designs were frequently weak. The conclusion made was that peer education programmes had some positive impact in reducing HIV transmission, through better knowledge of HIV, reduction in needle-sharing, and on condom use. However, in some target groups there was no significant impact on sexually transmitted infections (STI) suggesting that condom use was not always consistent.

Another systematic review ${ }^{7}$ of the evaluation of the effectiveness of peer education when compared to standard practice or no intervention, showed that there is little clear evidence of the effectiveness of peer education concerning HIV prevention, adolescent pregnancy prevention and sexual health promotion for young people in either developed, developing, or low- and middle-income countries.

The challenges of peer education programmes include the need for adequate resources to ensure consistency and quality of training for peer educators, balancing the time available for peer educators and their own needs and for the ability to be role models when they themselves are experiencing the same issues. $^{8}$

Peer education, particularly in South Africa and in other countries, appears to be successful in creating HIV and AIDS awareness but only moderately successful in effecting behavioural change. Peer education should not be regarded as a stand-alone strategy but as an adjunct to other interventions. ${ }^{9}$

\section{Condom use}

Condoms are $90-95 \%$ effective when used consistently, which is often not the case. If consistently used, condom users are 10 to 20 times less likely to become infected when exposed to the virus than inconsistent or non-users. ${ }^{10}$

Consequently, use of both male and female condoms has the potential to significantly reduce HIV infections and STIs. However, there is little correlation between the high rates of reported condom use and marginal reduction of STIs. ${ }^{11}$ In South Africa evidence suggests that the high rates of reported condom use may be a result of respondents giving socially desirable responses; reasons given included politeness, avoiding criticism and embarrassment. ${ }^{11}$ Another challenge to condom use is gender inequality and the threat of gender-based violence (GBV). ${ }^{11}$ There are also negative perceptions about the use of female condoms and of free public sector male condoms which were seen to be inferior in quality to those commercially available and socially marketed. ${ }^{12}$

Condoms are effective when properly used which is not the case. Condom distribution does not appear to be cost-effective. This strategy should be revisited with a view of how best to encourage consistent use.

\section{Voluntary medical male circumcision (VMMC)}

There is a substantial body of evidence that VMMC can reduce the risk of heterosexually acquired HIV infection in men by approximately $60 \%{ }^{7}$ The trials carried out in Kisumu, Kenya Akai District, and Uganda showed at least a 53\%, 51\% and 60\% reduction in risk of acquiring HIV infection, respectively. ${ }^{13}$ These results support findings published in 2005 from the South Africa Intervention Trial, sponsored by the French National Agency for Research on AIDS, which demonstrated at least a $60 \%$ reduction in HIV infection among men who were circumcised. ${ }^{13}$ WHO/ UNAIDS recommendations emphasise that VMMC should be considered an efficacious intervention for HIV prevention in countries and regions with heterosexual epidemics, high HIV and low male circumcision prevalence. ${ }^{13}$ Male circumcision provides only partial protection, and therefore should be only 
one element of a comprehensive HIV-prevention package which includes the provision of HIV testing and counselling services; treatment for sexually transmitted infections; the promotion of safer sex practices; the provision of male and female condoms when requested and promotion of their correct and consistent use. $^{12,13}$

\section{Prevention of mother-to-child transmission (PMTCT) of HIV}

PMTCT is perhaps the most dramatic and effective of HIVprevention interventions. Over the past 15 years South Africa has made remarkable progress towards reducing mother-tochild transmission (MTCT) of HIV. National MTCT-prevention (PMTCT) strategies have brought the risk of infant HIV infection down from $8 \%$ in 2008 to $3.5 \%$ in 2010, 2.6\% in 2012-2013 and an estimated $1.4 \%$ in 2015 ; in contrast to the $20-40 \%$ infant HIV infection risk measured in the absence of intervention strategies. ${ }^{14}$ PMTCT programmes also support safe childbirth practices and appropriate infant feeding, as well as providing infants exposed to HIV with virological testing after birth and during the breastfeeding period, ART for prevention and effective treatment. ${ }^{15,16}$

Undoubtedly, the PMTCT strategy is highly effective and it is suggested that it can be further improved by reducing the impact of stigma. ${ }^{16}$ Chiya et al. ${ }^{17}$ have found that PMTCT programmes must be strengthened by making the healthcare system more responsive to the experiences of healthcare workers and pregnant women to ensure effective implementation of changes to this programme.

\section{Pre-exposure prophylaxis (PrEP)}

Post-exposure prophylaxis (PEP) has been used for needlestick injury in health workers and also for those who have been subjected to sexual assault.

In contrast, pre-exposure prophylaxis (PrEP) is a daily course of ARVs such as oral tenofovir disoproxil fumarate (TDF) or oral TDF co-formulated with emtricitabine (TDF-FTC) and is currently being promoted as a strategy to protect HIV-negative people from HIV before potential exposure to the virus. Truvada ${ }^{\circledR}$ is a single combination pill of ARVs tenofovir and emtricitabine, and is currently the only drug approved for use as PrEP.18,19

A clinical trial among HIV-1-serodiscordant heterosexual couples has shown that, if taken daily and with strict adherence, PrEP is $45 \%$ effective for TDF and $75 \%$ for TDF-FTC and can reduce the chances of HIV infection; it is as high as $90 \%$ in those where it was shown that ARVs were present in the plasma. ${ }^{17}$ TDF-FTC combination was also effective in preventing HIV infection in gay men and other men who have sex with men. ${ }^{19}$ After the initial studies were completed and the results were known, researchers conducted further analysis and looked at a sample of people who were assigned to take TDF in the studies. It was only those participants who had TDF in their blood samples who were better protected against HIV. ${ }^{18,19}$ However, it is important to note that this 'post-hoc analysis' should be interpreted with caution since it was not one of the original goals of either study.
While it is suggested that perfect adherence is not essential, ${ }^{19}$ the effectiveness of PrEP is closely linked to adherence. If someone taking PrEP regularly misses daily doses, their risk of HIV infection will increase substantially. ${ }^{18,19}$ It is therefore important that any programme offering PrEP provides it as part of a combination package of prevention initiatives, based on the individual's circumstances together with support and advice on the importance of PrEP adherence. ${ }^{18,19}$

Understanding the rates at which people are able to adhere to PrEP, and addressing the barriers preventing adherence, will be crucial to the long-term success of this intervention. ${ }^{20}$

One study of three cities in the USA reported very different levels of PrEP adherence among men who have sex with men. In San Francisco, 52\% of participants took PrEP daily compared to $35 \%$ in Washington DC and just $13.5 \%$ in Miami. ${ }^{20} \mathrm{~A}$ further $27 \%$ of those from Miami, $18 \%$ from Washington and $4 \%$ from San Francisco only took two doses a week, offering about $70 \%$ protection (compared to over $90 \%$ if taken daily). Moreover, $11 \%$, $2 \%$ and $4 \%$ respectively took just one dose a week, offering very little protection; and $4.5 \%, 2 \%$ and $0 \%$ of participants had no detectable tenofovir in their blood. ${ }^{20}$ Without adherence, PrEP presents enormous challenges and should only be provided to those who have the motivation to use it properly.

Stigma associated with daily PreP is also a major challenge. PrEP may be associated or perceived to be associated with high-risk sexual activity and with other stigmas such as homosexuality, sex work, and/or drug use. ${ }^{21}$

Another of the drawbacks of this approach to HIV prevention is the not inconsiderable adverse effects, especially when given to HIV-negative men and women. ${ }^{18-20}$ Also, a potential disadvantage of PrEP is the generation of drug-resistant viruses and the resulting loss of treatment options. ${ }^{19,20}$

Current studies suggest that the PreP studies are a work in progress and much needs to be done to determine its effectiveness as a strategy and its cost-effectiveness. The consensus view in the literature is that it would seem prudent to only recommended PrEP for certain key affected populations such as sex workers, certain vulnerable women, men who have sex with men and people who inject drugs.

It should be noted that while PrEP can provide very effective protection against HIV, it does not provide protection against other STIs and blood-borne illnesses such as Hepatitis C, syphilis, and gonorrhoea. ${ }^{19,20}$

\section{Microbicides}

Microbicides are gels or creams containing antiretroviral drugs that are applied to the vagina to prevent HIV infection. Vaginal microbicides could be relatively effective, as long as they are used consistently and correctly. One study, the CAPRISA 004 trial $^{22}$ in South Africa, observed after 30 months, 39\% fewer infections generally and 54\% among women who were highly adherent, but its findings have not been replicated ${ }^{23,24}$ and major ethical concerns have been expressed concerning these studies. ${ }^{24}$ 
Apart from ethical considerations, these studies show that the main challenge with microbicides is adherence. The challenge is in creating a product that women who are at high risk of HIV infection are able to use regularly and consistently. In this respect, the issues for microbicides and PrEP are comparable. In fact, a microbicide gel is essentially a different way to deliver PrEP and is sometimes referred to as 'topical PrEP'.

In 2016, two large clinical trials seem to have addressed the challenges developing a vaginal ring that is used monthly.

The Ring Study, ${ }^{25}$ which was undertaken in South Africa and Uganda, and ASPIRE, ${ }^{26}$ conducted in Malawi, South Africa, Uganda and Zimbabwe, found that use of a monthly vaginal ring containing the antiretroviral drug, dapivirine, reduced rates of HIV acquisition by around one-third overall. In both studies, women aged over 21 used the ring more consistently and so more women in this age group were protected from HIV. However, there was little impact on HIV incidence in women aged $18-21$ as this age group was less likely to consistently adhere to use of the ring.

The HOPE trial ${ }^{27}$ (follow-up of the ASPIRE study), involving around 1300 women respectively in Malawi, South Africa, Uganda and Zimbabwe, found a 54\% reduction in HIV risk for dapivirine ring users, compared to non-users. Similarly, the DREAM trial ${ }^{28}$ which took place in South Africa and Uganda and involved 900 women, showed a $54 \%$ reduction in new HIV infections. These studies are the first vaginal ring studies to report above $50 \%$ efficiency.

Apart from the risk of developing resistance, the vaginal ring appears to be effective when adherence is high, particularly in older women.

\section{Treatment as prevention (TasP) for HIV}

Treatment as prevention (TasP) refers to HIV-prevention methods and programmes that use ART to decrease the risk of HIV transmission.

Evidence has now shown that individuals on consistent, effective ART with an undetectable viral load cannot transmit HIV to others. ${ }^{27,28}$ With consistent adherence, there is convincing evidence of the effectiveness of ART in prevention and it can be used as a public health intervention as well as a patient-specific strategy. ${ }^{29}$

The seminal HPTN $052^{30}$ study showed the personal and public health benefits of early treatment in HIV prevention. The study, involving 1763 discordant couples, found that early initiation of ART in the HIV-positive partner reduced cases of onward transmission to the HIV-negative partner by $96 \%$, compared to delayed treatment. Early treatment also resulted in $41 \%$ fewer adverse health events for the person living with HIV, compared to those not receiving treatment. A number of follow-up studies have also reported significant reductions in HIV transmission through early initiation of treatment, with new infections averted as a result. ${ }^{31,32}$ In 2014, the PARTNER study, 32 in which more than 1000 couples were enrolled, found no transmissions within mixed-status couples when the viral load of the positive partner was undetectable. The four-year study conducted across 14 European countries, which observed mixed-status couples where the viral load of the HIV-positive partner was undetectable, found zero transmissions after couples had sex 58000 times without a condom. The study, which included both heterosexual and gay couples, provides good evidence for the effectiveness of TasP. ${ }^{32}$ This evidence for the effectiveness of TasP has led to new World Health Organization (WHO) guidelines for a 'test and treat' or 'treat all' strategy - increasing testing and treatment coverage by initiating all people diagnosed with HIV on ART immediately, regardless of their CD4 count or viral load. This approach seeks to decrease community viral load and reduce the rate of new HIV infections. $^{33}$

This is a key cornerstone of UNAIDS' $90-90-90$ targets to end AIDS as a major public health problem by 2030 (90\% of all people living with HIV know their HIV status, $90 \%$ of all people diagnosed are on ART, $90 \%$ of all people on ART are virally suppressed). ${ }^{34}$ The WHO guidelines ${ }^{33}$ indicate that focus should be on specific population groups in whom the prevention impact is expected to be greatest such as serodiscordant couples, pregnant women, and gay couples. ${ }^{33}$

Undoubtedly TasP should be considered as a key element of HIVprevention strategies and as a major contributor to the solution to ending the HIV epidemic.

However, TasP has had mixed results in Africa. The ANRS 12249 trial $^{35}$ was the first of five large-scale randomised trials that took place in rural KwaZulu-Natal looking at the benefits of a universal test and treat for public health, rather than for the individual or their partners.

Despite increasing access to HIV testing and getting people who were on treatment virally suppressed, the results revealed that those diagnosed often did not link to medical facilities, or took many months to do so because of challenges experienced in accessing clinics. Only $49 \%$ of people diagnosed ultimately started treatment. This challenge of linking to care for test and treat reduced the number of people who went on to achieve an undetectable viral load to barely $42.4 \%$ of the population, reducing any possible population-level HIV-prevention benefits. A mathematical modelling study estimated that the implementation of universal voluntary HIV testing and immediate treatment initiation for adults over 15 years of age would take more than 50 years to decrease HIV prevalence to $1 \%$ in South Africa. ${ }^{36}$ However, other trials testing the effectiveness of TasP for the general population in high HIV-prevalence settings showed more promising results. The HPTN 071 study (also known as 'PopART') was one of the largest ever HIV test and treat trials and involved around one million people in South Africa and Zambia between 2013 and 2018. ${ }^{37}$

Results from the study found new HIV infections were 30\% lower in communities where test and treat was introduced alongside other proven HIV-prevention measures, compared to communities that received standard care. However, researchers found that treatment coverage was lower among young people (aged 29 and under) and among men. HIV treatment and 
prevention programmes will need to address these coverage gaps to realise the full potential of test and treat. ${ }^{37}$ Additional evidence from Uganda suggests that test and treat is having a significant impact on the number of people accessing HIV treatment. Uganda introduced test and treat in 2017. The number of men beginning treatment increased by 20000 over a single year (from 60000 in 2016 to 80000 in 2017), while the number of women increased by 31000 (from 107000 to 138000 ) over the same period. The gap between people being newly diagnosed with HIV and starting treatment has also significantly reduced, falling by $45 \%$ for men and $60 \%$ for women. ${ }^{37}$ The success of TasP is highly dependent upon people adhering to their treatment and linkage to health facilities..$^{37,38}$ Once treatment is initiated it should not be interrupted since incomplete viral suppression causes the more sensitive strains of HIV to be suppressed and the resistant strains, which are harder to treat, to become dominant. Testing, initiation of treatment and adherence are vital to the success of treatment as prevention. Adherence to treatment appears to be a global problem. Therefore, it is essential to provide support for PLHIV to ensure adherence. One study ${ }^{39}$ from Cape Town suggests that community-based approaches can improve retention rates. This study examined the success of community adherence clubs, meeting every two months, consisting of between 25 and 30 people, led by community health workers supported by nurses. The meetings provided group counselling, a brief symptom screening, and distribution of pre-packed ART. Group members were allowed to send a patient-nominated treatment supporter or 'buddy' to collect their ART at alternating group visits.

The study found this approach was highly effective, resulting in $94 \%$ of those taking part in the study adhering to treatment after a year. In addition, the adherence clubs were associated with a $67 \%$ reduction in the risk of people being lost to follow-up. ${ }^{39}$

Poor adherence and treatment interruptions also have the potential to lead to a significant increase in levels of HIV drug resistance (HIVDR) ${ }^{40}$ as seen in eastern and southern Africa. ${ }^{41}$

Consequently, attention must be paid to the HIVDR-contributing factors such as weak health systems, limited access to viral load testing and fewer resources for more expensive treatment regimens, all of which may undermine the benefits of a test and treat strategy. ${ }^{42}$

Steps must be in place in reducing the barriers for effective implementation of TasP.41,42

While TasP is an effective HIV-prevention strategy there are potential new challenges emerging around this intervention which need to be addressed, as listed below:

1. Different levels of readiness exist when it comes to engaging with HIV services; some are slower than others in moving from contemplating treatment to ensuring that those patients ready for treatment start immediately after testing, and subsequently stay on the treatment programme.
2. The capacity of health services and close access to facilities needs to be improved; that is, linkage to treatment needs to be improved.

3. Trust in healthcare providers must be improved and issues of stigma addressed.

4. Stigma, discrimination and other human rights violations deter people from seeking testing and treatment and also compromise their ability to adhere to ART.

\section{Conclusions}

In South Africa, HIV is a heterosexual infection that disproportionately affects younger women than men. It is this population where prevention strategies need the greatest focus, particularly in provinces with the highest prevalence and incidence rates, such as KwaZulu-Natal, Free Sate, Mpumalanga, Eastern Cape and Northwest. It is evident that no single strategy will be effective in HIV prevention and that a combination of strategies needs to be in place. Whatever approach is used, two factors are pertinent to achieving success. For prevention strategies to make an impact they need to target the most vulnerable populations and in provinces with the highest prevalence and incidence. This review has identified two major barriers for successful prevention of new infections. Adherence to any of the preventions is the single most important challenge. Where drug-based interventions are used, poor adherence may lead to resistance and potential adverse drug events, both of which can potentially limit the success of prevention strategies.

Behavioural change and abstinence are the obvious and most effective approaches to prevention. However, abstinence is not a realistic strategy. In its absence safe sex through condom use has proven to be effective. However, as shown in this review, for a host of reasons condom use has limited success, particularly the use of female condoms. For many women, negotiating safe sex can be a challenge due to gender inequality and gender-based violence.

The widespread availability of condoms is not cost-effective and distribution should perhaps be demand-based and available through retail pharmacies and public sector clinics. VMMC has also proved to have significantly reduced the risk of HIV infection in men and is actively promoted in South Africa with some success; continued effort needs to be maintained to improve uptake.

Use of microbicidal gels and rings, while only partially effective, are fraught with mainly ethical concerns, particularly where the male partner is unaware that his partner is using the gel or ring. Adherence and development of resistance are also of concern. The vaginal ring overcomes some of the problems of adherence and partner awareness, particularly in older women, yet development of resistance may be a concern.

Certain partially effective strategies, such as PreP, for reasons of low adherence, stigma, and adverse effects should be targeted for vulnerable populations such as sex workers and vulnerable young women who have sex particularly with older men. Perhaps PreP should be offered on request through community- 
based programmes. Cost-effective analysis of biomedical HIV interventions in South Africa suggests that VMMC, vaginal microbicides and oral PrEP could be cost-effective only for certain vulnerable populations. ${ }^{44}$ However, PreP appears to be the least cost-effective. ${ }^{43}$

Undoubtedly, one of the most successful and most costeffective $^{44}$ interventions is PMTCT in the prevention of HIV transmissions during birth and subsequently.

Key to the success of any prevention strategy is "knowing your status" and this forms the cornerstone of the South African Department of Health's national HIV intervention programme. If all HIV-positive persons are treated with ARVs regardless of CD4 counts or viral load, then TasP would be the most effective and cost-effective ${ }^{44}$ prevention measure. Critical for the success of this approach is adherence. Systemic factors such as financial resources, ARV availability, linkage to facilities, trained healthcare professionals and community support structures are also critical contributing factors for success.

Ultimately, a combination of approaches for the specifically targeted populations that include condom use, VMMC and TasP has been shown to be cost-effective. Focus should be on strategies that are cost-effective such as VMMC, PMTCT and TasP. Additional strategies that involve PreP should be considered for vulnerable populations. Underpinning all this is community involvement, particularly community health workers linked to clinics and patient support groups.

\section{References}

1. TB Facts.org: HIV in South Africa - High burden districts, key populations, gay men. https://www.tbfacts.org/hiv-south-africa/ Accessed 25 May 2019.

2. HIV Statistics: https://www.tbfacts.org/hiv-statistics-south-africa/ Accessed 18 September 2019.

3. National Research Council; Division of Behavioral and Social Sciences and Education; Commission on Behavioral and Social Sciences and Education, Preventing and Mitigating AIDS in Sub-Saharan Africa: Research and Data Priorities for the Social and Behavioural Sciences, 1996 (Chapter 5) Barney Cohen and James Trussell, Editors. https://www.nap.edu/read/5177/ chapter/8\#165. Accessed 25 May 2019.

4. Health Sector HIV Prevention Strategy 2016. Department of Health Users/ MOODLEYI15/ Downloads/health\%20sector\%20hiv\%20prevention\%20for\%20 print\%20(7).pdf Accessed 18 September 2019.

5. Https://www.avert.oreg/professionals/hiv around the world/sub-sub-saharanafrica/south africa. Accessed 18 September 2019.

6. National Research Council (US) Panel on Data and Research Priorities for Arresting AIDS in Sub-Saharan Africa. Cohen B, Trussell J, editors. Preventing and Mitigating AIDS in Sub-Saharan Africa: Research and Data Priorities for the Social and Behavioural Sciences. Washington (DC): National Academies Press (US); 1996. 1, Introduction. Available from: https://www.ncbi.nlm.nih.gov/books/ NBK232821. Accessed 25 May 2019.

7. Medley A, Kennedy CK, O'Reilly K, Sweat M. Effectiveness of peer education interventions for HIV prevention in developing countries: A systematic review and meta-analysis. 2009; AIDS Educ Prev 21:181-206. doi: 10.1521/ aeap.2009.21.3.181.

8. Tolli MV. Effectiveness of peer education interventions for HIV prevention, adolescent pregnancy prevention and sexual health promotion for young people: a systematic review of European studies. Health, 2012: Education Research 2;904-913 (https://doi.org/10.1093/her/cys055.)

9. Peer education and HIV/AIDS: Concepts, uses and challenges. Joint United Nations Programme on HIV/AIDS(UNAIDS) 1999: ( www.unaids.org resources ) documents > 20001019_jc291-peereduc_en [Accessed 18 September 2019].

10. Condoms for HIV prevention - WHO https://www.who.int > hiv > topics > condoms en Accessed 18 September 2019.
11. Van Loggerenberg F, Dieter AA, Sobieszczyk ME, et al. 2012: HIV prevention in high-risk women in South Africa: Condom use and the need for change. PLoS ONE.; 7(2): e30669. doi:10.1371/journal.pone.0030669.

12. Mags E, Beksinska M, Smit JA, Mantell JE. 2012. Progress and challenges to male and female condom use in South Africa. Sex Health. 51-58. doi: 10.1071/ SH11011.

13. New data on male circumcision and HIV prevention: Policy and programme implications. 2007.

14. Https://www.who.int/hiv/pub/malecircumcision/research implications/en/ : ISBN 978924 159598. Accessed 25 May 2019. Goga A, Chirinda NK, Ngoma K, et al. Closing the gaps to eliminate mother-to-child transmission of HIV (MTCT) in South Africa: Understanding MTCT case rates, factors that hinder the monitoring and attainment of targets, and potential game changers. 2018: S Afr Med J; 108, (3 Suppl 1);S17-S24. (DOI:10.7196/).

15. Prudden HJ, Hamilton M, Foss AM, et al. Can mother-to-child transmission of HIV be eliminated without addressing the issue of stigma? Modelling the case for a setting in South Africa. PLoS ONE 20172 12; (e0189079. https://doi.org/10.1371/ journal.pone.0189079\}

16. Chiya HW, Naidoo JR, Ncama BP, et al. Stakeholders' experiences in implementation of rapid changes to the South African prevention of mother-to-child transmission programme. Afr J Prm Health Care Fam Med. 2017:10(1):a1788. https:// doi.org/10.4102/phcfm. v10i1.1788.

17. Baeten JM, Donnell D, Ndase PH, et al, for the Partners PrEP Study Team* Antiretroviral prophylaxis for HIV prevention in heterosexual men and women: $\mathrm{N}$ Engl J Med.2012: :367;399-410 (DOI: 10.1056/NEJMoa1108524).

18. McCormack S, Dunn DT, Desai M, et al. ON: Pre-exposure prophylaxis to prevent the acquisition of HIV-1 infection (PROUD): effectiveness results from the pilot phase of a pragmatic open-label randomised trial. Lancet. 2015: 387;53-60.( doi: 10.1016/50140-6736(15)00056-2. Epub $2015 \mathrm{Sep})$.

19. Grant RM, Lama JR, Anderson PL, et al. Preexposure chemoprophylaxis for HIV prevention in men who have sex with men. N Engl J Med. 2010:363;2587-2599. (doi: 10.1056/NEJMoa1011205. Epub 2010 Nov 23. https://www.ncbi.nlm.nih. gov/pmc/articles/ PMC3079639/).

20. Van Damme L, Corneli A, Ahmed K, et al, for the FEM-PrEP Study Group* Preexposure prophylaxis for HIV infection among African women. N Engl J Med. 2012:367;:411-422 (DOI: 10.1056/NEJMoa1202614).

21. Abdool Karim Q, Abdool Karim SS, Frohlich, et al. CAPRISA 004 Trial Group: Effectiveness and safety of tenofovir gel, an antiretroviral microbicide, for the prevention of HIV infection in women. Science. 2010:5996; 1168-74 ( doi: 10.1126/science.1193748. Epub 2010 Jul 19. Erratum in: Science. 2011 Jul 29;333(6042):524. PubMed PMID: 20643915; PubMed Central PMCID: PMC3001187).

22. Delany-Moretlwe S, Lombard C, Baron D, et al. Tenofovir $1 \%$ vaginal gel for prevention of HIV-1 infection in women in South Africa (FACTS-001): a phase 3 , randomised, double-blind, placebo-controlled trial. Lancet Infectious Disease. 2018:18;(11):1241-1250.

23. Jeanne M, Marrazzo J, Ramjee G, et al, for the VOICE Study Team: Tenofovirbased preexposure prophylaxis for HIV infection among African women. $N$ Engl J Med. 2015:372;509-518 (DOI: 10.1056/NEJMoa1402269).

24. Moodley K. 2007: Microbicide research in developing countries: have we given the ethical concerns due consideration? BMC Medical Ethics. 8;10 (https://doi. org/10.1186/1472-6939-8-10).

25. Nel A, Van Niekerk N, Kapiga S, et al, 201 for the Ring Study Team* 2016: Safety and efficacy of a dapivirine vaginal ring for HIV prevention in women. $\mathrm{N}$ Engl $\mathrm{J}$ Med. 375;2133-2143. (DOI:10.1056/NEJMoa 1602046).

26. Taha E, Jones J, Mayo A, Scheckter R, et al, for the MTN-020-ASPIRE Study Team. Use of a vaginal ring containing dapivirine for HIV-1 prevention in women. $\mathrm{N}$ Engl J Med. 2016:375;2121-2132.

27. Baeten J, Rosenberg Z. High uptake and reduced HIV-1 incidence in an open-label trial of the dapivirine vaginal ring. Conference on retroviruses and opportunistic infections, Boston. Mar. 7, 2018.Vaginal ring appears to cut women's HIV rate in half in new study (HOPE Study) (https://www.ipmglobal.org/ content/vaginal-ring-appears-cut-womens-hiv-rate-half-new-study).

28. Nel A, Van Niekerk N, Kapiga S, et al. 2016. Safety and efficacy of a dapivirine vaginal ring for HIV prevention in women. $\mathrm{N}$ Engl J Med. 2016:1;375(22);2133-2143.

29. UNAIDS (12 May, 2011): Groundbreaking trial results confirm HIV treatment prevents transmission of HIV. Accessed 25 May 2019.

Full list of references available on request. 
30. Cohen MS, Chen YQ, McCauley M, et al. HPTN 052 Study Team. Prevention of HIV-1 infection with early antiretroviral therapy. The New England Journal of Medicine, 2011. 365(6)4; 93-505. doi:10.1056/NEJMoa1105243.

31. Das $M, C h u$ PL, Santos G-M, et al. Decreases in community viral load are accompanied by reductions in new HIV infections in San Francisco. PLoS ONE 2010: 5(6); e11068. https://doi.org/10.1371/journal.pone.001106.

32. Rodger AJ, Cambiano V, Bruun T, et al. (2016) Sexual activity without condoms and risk of HIV transmission in serodifferent couples when the HIV-positive partner is using suppressive antiretroviral therapy. The Journal of the American Medical Association. 2016:316;171-181.

33. World Health Organization (WHO) 2015: Guideline on when to start antiretroviral therapy and on pre-exposure prophylaxis for HIV [pdf].

34. UNAIDS 2014: 90-90-90: An ambitious treatment target to help end the AIDS (2) epidemic [pdf].

35. Iwuji CC, Orne-Gliemann J, Larmarange J, et al. Uptake of home-based HIV testing, linkage to care, and community attitudes about ART in rural KwaZuluNatal, South Africa: Descriptive results from the first phase of the ANRS 12249 TasP Cluster-Randomised Trial. PLoS.:Med 2016: 13(8); e1002107. doi:10.1371/ journal.pmed.1002107.

36. Granich RM, Gilks CF, Dye C, et al. Universal voluntary HIV testing with immediate antiretroviral therapy as a strategy for elimination of HIV transmission: a mathematical model. Lancet 2009:373;48-57 Published Online November 26, 2008 DOI:10.1016/S0140-6736(08)61697-9.

37. Cori A, Ayles H, Beyers N, et al. HPTN 071 (PopART): A cluster-randomized trial of the population impact of an HIV combination prevention intervention including universal testing and treatment: mathematical model. PLoS ONE. 2014:9(1;: e84511. doi:10.1371/journal.pone.0084511.

38. UNAIDS 2018: Test and treat: https://www.unaids.org/en/resources/presscentre/ featurestories/2018/ april/test-and-treat-showing-results-in-Uganda-andZambia Accessed 30 May 2019.

39. Grimsrud A, Sharp J, Kalombo C, et al. Implementation of community-based adherence clubs for stable antiretroviral therapy patients in Cape Town, South Africa. Journal of the International AIDS Society 2015:18;19984 http://www. jiasociety. org/index.php/jias/ article/view/19984 | http://dx.doi.org/10.7448/ IAS.18.1.19984.

40. Shelton JD. HIV/AIDS. ARVs as HIV prevention: a tough road to wide impact. Science. 2011:334(6063);1645-6. doi: 10.1126/science.1212353.

41. UNAIDS 2018: Miles to go: global update 2018. https://www.unaids.org/ en/20180718_GR2018.

42. Skovdal M, Nyamukapa C, Wamoyi J, et al. Poster presentation: Opportunities and challenges for 'test-and-treat': Insights from eastern and southern Africa: World AIDS 21 Durban 2016.

43. Long EF, Stavert RR. Portfolios of biomedical HIV interventions in South Africa: A cost-effectiveness analysis. J Gen Intern Med. 2013: 28;:1294-1301,DOI: 10.1007/ s11606-013-2417-1.

44. Margo M, Jacobsen MM, Walensky RP. Modeling and cost-effectiveness in HIV prevention. Curr HIV/AIDS Rep. 2016:13(1);64-75. doi:10.1007/ s11904-016-0303-2. 